\title{
Superconductivity in the two-band Hubbard model
}

\author{
K P JAIN, R RAMAKUMAR and C C CHANCEY* \\ Laser Technology Research Programme. Indian Institute of Technology, New Delhi 110016, \\ India. \\ *Department of Physics, Amherst College, Amherst. MA 01002, USA

\begin{abstract}
We present a study of superconducting correlations in a two-band Hubbard model with a wide band strongly hybridized with a narrow band in which an attractive on-site interaction is operating. The narrow band pairs can induce superconducting correlations in the wide band through hybridization interaction. A generalized gap function for the induced wide-band pairing is obtained and its properties in the intermediate interaction region are analysed. Relevance of the results to high- $T_{\mathrm{c}}$ superconductors is briefly discussed.
\end{abstract}

Keywords. Two-band Hubbard model; correlations.

\section{Introduction}

An attractive on-site interaction can arise in many narrow band materials due to bipolaronic mechanism, excitonic mechanism, coupling of electrons to other electronic substems in a solid or abnormal bond configurations in non-simple metals etc. (Little 1964; Moizhes and Drabkin 1983). In the strong coupling limit this attraction leads to the formation of localized on-site pairs which can induce superconductivity in the wide band of carriers through the hybridization coupling (Robaskiewicz et al 1987). In this paper we study this pairing process in the intermediate onsite attraction regime.

\section{Hamiltonian and superconducting gap functions}

Our starting off point is the following two-band Hamiltonian of hybridized wide and narrow bands

$$
H=H_{d}+H_{p}+H_{p d}
$$

where the Hamiltonian in the narrow $d$ - and wide $p$-bands respectively are,

$$
\begin{aligned}
& H_{d}=\sum_{i \sigma} \varepsilon_{d} n_{i \sigma}+\sum_{i j \sigma} T_{i j}^{d} c_{i \sigma}^{+} c_{j \sigma}+I \sum_{i} n_{i \dagger} n_{t !}, \\
& H_{p}=\sum_{i \sigma} \varepsilon_{p} n_{i \sigma}^{p}+\sum_{i j \sigma} T_{i j}^{p} p_{i \sigma}^{+} p_{j \sigma}
\end{aligned}
$$

and

$$
H_{p d}=\sum_{i j \sigma} h_{i j}\left(p_{i \sigma}^{+} c_{j \sigma}+c_{j \sigma}^{+} p_{i \sigma}\right)
$$

is the term coming from hybridization. In the above equations $I$ is the on-site attraction between the narrow band electrons and $h$ is the hybridization matrix element. Other

*For correspondence 
symbols have their usual meaning. To go beyond the Hartree-Fock mean-field regime, we introduce $d$ (Hubbard and Jain 1968; Jain et al 1989): $d_{i \sigma}^{\alpha}=n_{i-\sigma}^{\alpha} c_{i \sigma}$, where $n_{i-\sigma}^{+}=n_{i-\sigma}$ and $n_{i-\sigma}^{-}=1-n_{i-\sigma}$. Now, the Heisenberg equations of motion for $d_{i \sigma}^{\alpha}$ and $p_{i \sigma}$ respectively are:

$$
\begin{aligned}
i d_{i \sigma}^{\alpha}= & \varepsilon_{\alpha} d_{i \sigma}^{\alpha}+\sum_{j \neq i} T_{i j}^{d} n_{i-\sigma}^{\alpha} c_{j \sigma}+\zeta^{\alpha} S_{i-\sigma} c_{i \sigma} \\
& -\sum_{j} h_{i j}\left[\xi^{\alpha}\left(p_{j-\sigma}^{\dagger} c_{i-\sigma} c_{i \sigma}+c_{i-\sigma}^{\dagger} c_{i \sigma} p_{j-\sigma}\right)-n_{i-\sigma}^{\alpha} p_{j \sigma}\right] \\
i \dot{p}_{i \sigma}= & \varepsilon_{p} p_{i \sigma}+\sum_{k \neq i} T_{i k}^{p} p_{k \sigma}+\sum_{j} h_{i j} c_{j \sigma},
\end{aligned}
$$

where

$$
S_{i-\sigma}=\sum_{j \neq i} T_{i j}^{d}\left(c_{i \sigma}^{\dagger} c_{j \sigma}-c_{j \sigma}^{\dagger} c_{i \sigma}\right) ; \varepsilon_{+}=I+\varepsilon_{d} ; \varepsilon_{-}=\varepsilon_{\mathrm{d}} \text { and } \xi^{+}=-\xi^{-}=1 .
$$

Now, we introduce the following approximations:

$$
\begin{aligned}
& p_{j-\sigma}^{\dagger} c_{i-\sigma} c_{i \sigma} \simeq\left\langle c_{i-\sigma} c_{i \sigma}\right\rangle p_{j-\sigma}^{\dagger}+\left\langle p_{j-\sigma}^{\dagger} c_{i-\sigma}\right\rangle c_{i \sigma}, \\
& n_{i-\sigma}^{\alpha} p_{j \sigma} \simeq\left\langle n_{i-\sigma}^{\alpha}\right\rangle p_{j \sigma} ; c_{i-\sigma}^{\dagger} c_{i \sigma} p_{j-\sigma} \simeq\left\langle c_{i-\sigma}^{\dagger} p_{j-\sigma}\right\rangle c_{i \sigma}, \\
& n_{i-\sigma}^{\alpha} c_{j \sigma} \simeq\left\langle n_{i-\sigma}^{\alpha}\right\rangle c_{j \sigma}+\xi^{\alpha}\left\langle c_{i-\sigma} c_{j \sigma}\right\rangle c_{i-\sigma}^{\dagger}
\end{aligned}
$$

and

$$
S_{i-\sigma} c_{i \sigma} \simeq\left\langle S_{i-\sigma}\right\rangle c_{i \sigma}+\sum_{j \neq i}\left\langle c_{j-\sigma} c_{i \sigma}\right\rangle T_{i j} c_{i-\sigma}^{\dagger}-\sum_{j \neq i}\left\langle c_{i-\sigma} c_{i \sigma}\right\rangle T_{i j}^{d} c_{j-\sigma}^{\dagger} .
$$

Note that direct pairing amplitude in the wide band is not included in the above approximations. Further, fluctuations in all order parameters and spin-flip amplitudes are not considered. Then, the above decoupling restricts the entire treatment to the weak to intermediate coupling regime (i.e. $|I| \leqslant W_{d}$ where $W_{d}$ is the narrow bandwidth. Inclusion of spin fluctuations is straightforward in the weak coupling limit (Jain and Chancey 1989). Using these approximations we have solved the simultaneous equations (5) and (6) after recasting them in terms of the Hubbard sub-band operators $\tilde{D}_{k \sigma}^{\mu}=\varepsilon_{k}^{-1} N_{k \mu} D_{k \sigma}^{\mu}$ where $D_{k \sigma}^{\mu}=\sum_{\alpha} d_{k \sigma}^{\alpha} /\left(E_{k \mu}-\varepsilon_{\alpha}\right)$. Here $N_{k \mu}^{-1}=\sum_{\alpha} n_{\alpha} /\left(E_{k \mu}-\varepsilon_{\alpha}\right)^{2}$ and $E_{k \mu}$ are the Hubbard sub-band energies. We obtain two effective equations for $p$ and $d$-band,

$$
i \dot{p}_{k \sigma}(t)-\tilde{\tilde{\varepsilon}}_{k}^{p} p_{k \sigma}(t)-\tilde{\tilde{\Delta}}_{k} p_{-k \sigma}^{\dagger}(t)=0
$$

and

$$
i \tilde{D}_{k \sigma}^{\mu}(t)-\bar{E}_{k \mu} \tilde{D}_{k \sigma}^{\mu}(t)-\tilde{D}_{-k-\sigma}^{\dagger \mu}(t)=0
$$

where

$$
\begin{aligned}
& \tilde{\varepsilon}_{k}^{p}=\varepsilon_{k}^{p}+O(h), \quad \tilde{\tilde{\varepsilon}}_{k}^{p}=\tilde{\varepsilon}_{k}^{p}-h_{k}^{2} \sum_{\mu} N_{k \mu} \varepsilon_{k}^{-\tilde{\varepsilon}^{2}} /\left(E_{k \mu}-\tilde{\varepsilon}_{k}^{p}\right), \\
& \tilde{E}_{k \mu}=E_{k \mu}+O(h), \quad \tilde{E}_{k \mu}=\tilde{E}_{k \mu}+\varepsilon_{k}^{\tilde{d}^{2}} N_{k \mu} h_{k}^{2} \sum_{y} 1 /\left(E_{k \nu}-\tilde{\varepsilon}_{k}^{p}\right) .
\end{aligned}
$$

The two generalized superconducting gap functions for wide and narrow bands are respectively,

$$
\tilde{\tilde{\Delta}}_{k}^{p}=h_{k}^{2} \sum_{\mu} \frac{\varepsilon_{k}^{-1} N_{k \mu} T_{k \mu} \Delta}{\left(\tilde{E}_{k \mu}^{2}+\bar{\Delta}_{k \mu}^{2}\right)^{1 / 2}+\tilde{\varepsilon}_{\mu}^{p}},
$$


and

$$
\bar{\Delta}_{k \mu}^{d}=\bar{\Delta}_{k \mu}+h_{k}^{2} \sum_{y} \frac{\varepsilon_{k}^{d} N_{k \mu} T_{k \mu} \Delta}{\left(\bar{E}_{k v}^{2}+\bar{\Delta}_{k v}^{2}\right)-\tilde{\varepsilon}_{k}^{p}}
$$

where $\bar{\Delta}_{k \mu}=\left\{\Delta+2 \varepsilon_{k}^{d} \sum_{k^{\prime}} \varepsilon_{k^{\prime}}^{d}\left\langle c_{-k^{\prime}-\sigma} c_{k^{\prime} \sigma}\right\rangle\right\} T_{k \mu} N_{k \mu}$ is the function in the decoupled $d$-band (i.e. for $h=0$ ) and $\Delta=\sum_{k}\left\langle c_{k \sigma} c_{-k-\sigma}\right\rangle$. Expressing $\Delta$ in terms of the Hubbard subband operators it is straightforward to obtain the self-consistency conditions for the gap functions and the chemical potential.

\section{Properties of the generalized gap function}

The underlying physical idea in this treatment is that superconducting pair formation in the narrow band resulting from the on-site attractive interaction can induce pairing in the wide band of nearly free carriers through the hybridization interaction and consequently a superconducting gap opens up in the single particle excitation spectrum of the wide-band electrons. Note that there is no pairing amplitude in the wide band. Then the indirect hybridization induced pair formation is naturally second order in hybridization. In the limit $h_{k} \rightarrow 0, \tilde{\tilde{\Delta}}_{k}$ vanishes. In the $I \ll W$ limit on-site $I$ becomes the weak average pairing potential, Hubbard sub-bands lose their individual character, and consequently many-body effects do not arise, we get a BCS-like result for $\widetilde{\Delta}_{k}$, implying weak pairing. But beyond this weak coupling regime, higher order correlations significantly modifies the gap function in the narrow band and consequently changes the entire character of the wide-band pairing. The entire Fermi sea is involved in the pairing and this implies a small coherence length in this regime. In this limit $0 \geqslant E_{k 1} \geqslant 0\left(\varepsilon_{k}^{d}\right)$ and $-W \geqslant E_{k 2} \geqslant-W+0\left(\varepsilon_{k}^{d}\right)$, which leads to $\left|\bar{\Delta}_{k 1,2}\right|=\left(\Delta+2 \tilde{\varepsilon}_{0}^{d} \varepsilon_{k}^{-1}\right.$ $O\left(W_{d}\right)$, so that

$$
\tilde{\Delta}_{k}=h_{k}^{2} \varepsilon_{k}^{-1} \Delta\left(\frac{1}{\left(E_{k 1}^{2}+\bar{\Delta}_{k 1}^{2}\right)^{1 / 2}+\tilde{\varepsilon}_{k}^{p}}-\frac{1}{\left(\tilde{E}_{k 2}^{2}+\bar{\Delta}_{k 2}^{2}\right)^{1 / 2}+\tilde{\varepsilon}_{k}^{p}}\right) O\left(W_{d}\right) .
$$

It is seen that indirect pairing gap in the wide band is proportional to the width of the narrow band. The pairing amplitude will also strongly depend on the particle number in the narrow band. It will increase with number density for $n_{d}<1$ and go through a maximum near $n_{d}=1$ and decrease beyond half-filling.

\section{Conclusions}

We conclude with a brief discussion on the relevance on this model to high $T_{c}$ superconductors. Recent experimental studies (Sarma et al 1987, 1988) have concluded that the carriers in the hole superconductors are in the oxygen $p$-band which is weakly correlated and a realistic model to understand these systems is a two-band model of strongly hybridized $\mathrm{Cu} d$ - and $\mathrm{O} p$-bands (Emery 1987; Varma et al 1987). The direct pairing amplitude of $p$-holes is understood to be negligible and the high- $T_{c}$ results from the interaction of this band with the narrow band. Then our results can be applied to these systems. An attractive interaction san arise from bipolaronic, excitonic or by 
polarization waves etc. in the narrow band and this can induce pairing in $p$-band as discussed. An important drawback of this approach, then, is that pairs exist even in the undoped material. But, if we assume that attractive on-site coupling is operative only below $T_{c}$, the normal state could either be Mott-Hubbard or charge transfer insulator depending on the ratio $U /\left|\left(\varepsilon_{p}-\varepsilon_{d}\right)\right|$, where $U$ is on-site repulsion. This model is also relevant to superconductivity in heavy Fermion systems. Assumption of finite band energy, perhaps, makes it more closer to these systems in view of the poor, but finite, conductivity in the normal state.

\section{References}

Emery V J 1987 Phys. Rev. $\mathbf{5 8} 2794$

Hubbard J and Jain K P 1968 J. Phys. 11650

Jain K P et al 1990 Physica $\mathbf{C 1 6 8} 297$

Jain K P and Chancey C C 1989 Phys. Rev. B39 9049

Little W A 1964 Phys. Rev. 134 A1416

Moishes B Ya and Drabkin I A 1983 Sov. Phys. - Solid State Phys. 251139

Robaskiewicz S et al 1987 Phys. Rev. B36 180

Sarma D D et al 1987 Phys. Rev. B36 2371

Sarma D D et al 1988 Solid State Commun. 62263

Varma C M et al 1987 Solid State Commun. 62981 